\title{
VACANCIES IN THE MOLECULAR CRYSTAL OF 2-(2'-HYDROXYPHENYL)BENZOTHIAZOLE
}

\author{
Y. Syetov \\ Oles Honchar Dnipro National University, Dnipro, Ukraine \\ e-mail: setov2003@yahoo.com
}

\begin{abstract}
Structure of molecular units is calculated for the periodic model corresponding to the crystal lattice of 2-(2'-hydroxyphenyl)benzothiazole with vacancies. 2-(2'-hydroxyphenyl)benzothiazole is a luminescent organic substance undergoing excited state intramolecular proton transfer. The calculations are performed with density-functional based tight-binding methods usding Van der Waals interaction empirical correction. It is found that the dihedral angles formed by benzothiazole and phenol parts of the molecules deviate in the vicinity of the vacancy. The vacancy provides enough space for non-planar conformation of the molecules in the ground state. At the same time the increase in energy of the periodic structure with the vacancies caused by appearance of the non-planar conformation is larger than the corresponding increase in the isolated molecule.
\end{abstract} method.

Keywords: molecular crystal, excited state proton transfer, defects, density-functional based tight-binding

Received 25.06.2021; Received in revised form 28.07.2021; Accepted 15.08.2021

\section{Introduction}

2-(2'-hydroxyphenyl)benzothiazole (HBT) (Fig. 1a) is an organic compound that exhibits photoinduced excited state intramolecular proton transfer. HBT has been studied for about 50 years as a model compound for ESIPT [1,2]. HBT is also tried for applications in optoelectronics including emitting layers of electroluminescent devices [3] and lasing material [4]. In the ground state the most stable structure is enol with an $\mathrm{OH} . . \mathrm{N}$ hydrogen bond. Other possible conformations of the molecule formed by rotation of the benzothiazole and phenol moieties as well as the $\mathrm{OH}$ bond demonstrate the much higher energy than the most stable structure implying their very low equilibrium concentration even at relatively high temperature [5]. In the excited state the keto structure with an O...HN hydrogen bond is the most stable and there is internal rotation in the isolated molecule. The internal rotation in the keto structure is considered to be the main path for radiationless transition to the ground state lowering quantum yield of luminescence [2]. In the experiments on HBT nanowires as an optically pumped laser medium amplified emission is observed at two wavelengths and interpreted as presence of planar and partially twisted keto structures [4].

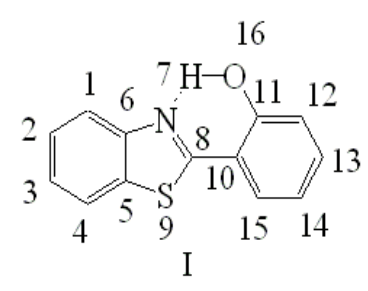

a

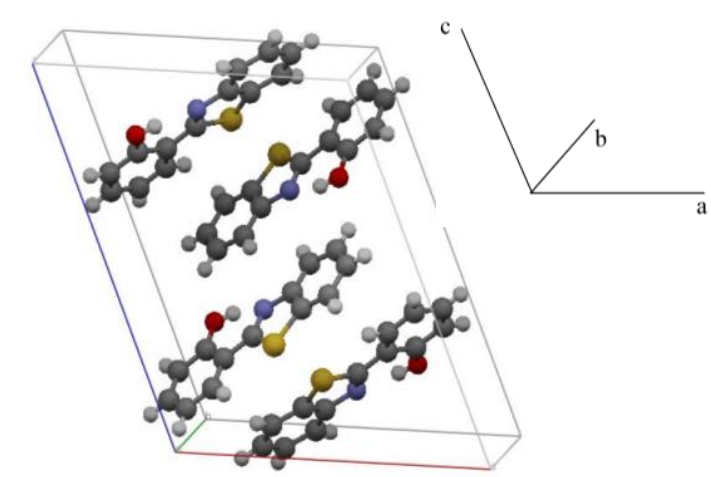

b

Fig. 1. Enol structure of molecule with atomic numbering (a) and a unit cell of a crystal of HBT (b). The unit cell is plotted according to the $\mathrm{X}$-ray data [7]. 
X-ray data show [6,7] that HBT forms monoclinic P2 1 /c structure class crystal with 4 molecules in a unit cell. (Fig. 1b). It is expected that crystal packing hinders the intramolecular rotation causing high quantum yield of luminescence in crystals. Theoretical calculations demonstrate that defects caused by different orientation as whole molecule as the benzothiazole or phenol fragments have high energy implying their low equilibrium concentration [5]. The calculations [5] and X-ray measurements [6,7] show that the enol structure of molecules of HBT in crystal is close to planar, the structure of the isolated molecules is predicted to be strictly planar.

Besides different orientation of the molecules in the crystal lattice the common point defects in molecular crystals are vacancies, that is absence of a molecule in the site [8]. The absence of the molecules causes deformation of the periodic structure in the vicinity of the vacancy.

In the present paper we evaluate effect of vacancies on the geometrical parameters of molecular units in the crystal of HBT.

\section{Calculations details}

Geometrical parameters of model structures of an HBT crystal with vacancies were calculated by density functional based tight-binding (DFTB) method with the third order correction and Van der Waals interaction correction implemented in the DFTB+ program package [9,10] as described in [11] for ideal crystal. As it is found for benzoxazole analogue 2-(2'-hydroxyphenyl)benzoxazole (HBO), this calculation method provides good correspondence of the predicted frequencies of low-frequency lattice vibrations for ideal crystal and positions of Raman bands observed in the experimental spectrum [11]. A supercell folding is used for the Brillouin zone sampling with the $2 \times 2 \times 2$ supercell. The periodic structures are optimized for the Brillouin zone center assuming the cell parameters be fixed to the values taken from the X-ray data [6]. The density functional theory (DFT) and time-dependent density functional theory (TDDFT) calculations for isolated molecular structures were also performed with the GAMESS program suite $[12,13]$ using the B3LYP functional and 6-31G(d,p) basis set.

The periodic model structure of the defect comprises 18 unit cells of the crystal obtained by translation along the lattice vectors $3 \mathbf{a}, 3 \mathbf{b}$ and $2 \mathbf{c}$ (Fig. 2). One molecule in this structure is removed to model the vacancy.

\section{Results and discussion}

The calculations reveal that there is a difference between the values of the dihedral angles $\mathrm{N}_{7}-\mathrm{C}_{8}-\mathrm{C}_{10}-\mathrm{C}_{11}$ and $\mathrm{S}_{9}-\mathrm{C}_{8}-\mathrm{C}_{10}-\mathrm{C}_{15}$ for the molecule in the vicinity of the vacancy and more distant molecules. The dihedral angles $\mathrm{N}_{7}-\mathrm{C}_{8}-\mathrm{C}_{10}-\mathrm{C}_{11}$ and $\mathrm{S}_{9}-\mathrm{C}_{8}-\mathrm{C}_{10}-\mathrm{C}_{15}$ describe mutual orientation of the benzothiazole and phenol moieties in the HBT molecule (see Fig. 1a). In the most molecules that do not constitute the first coordination sphere of the vacancy the values of the $\mathrm{N}_{7}-\mathrm{C}_{8}-\mathrm{C}_{10}-\mathrm{C}_{11}$ dihedral angles are about $6^{\circ}$. The value is close to that obtained for ideal crystal lattice [5]. In the vicinity of the vacancies the smallest value of the $\mathrm{N}_{7}-\mathrm{C}_{8}-\mathrm{C}_{10}-\mathrm{C}_{11}$ dihedral angle is $2.5^{\circ}$, the largest $-8.4^{\circ}$. The corresponding values of the $\mathrm{S}_{9}-\mathrm{C}_{8}-\mathrm{C}_{10}-\mathrm{C}_{15}$ dihedral angles are about $5.5^{\circ}, 2.0^{\circ}$ and $8.3^{\circ}$. For the isolated molecule the dihedral angles are about $0^{\circ}$.

DFT and TDDFT calculations of energy performed on the structures with the $\mathrm{N}_{7}-\mathrm{C}_{8}-$ $\mathrm{C}_{10}-\mathrm{C}_{11}$ angles of $6^{\circ}$ and $2.5^{\circ}$ extracted from the model show that the energy difference is less than $0.01 \mathrm{eV}$ both in the ground and first excited states. Consequently, energy of the transitions from the ground and excited states differ negligibly. The variation of the dihedral angle does not change the energy of the molecules caused by intramolecular forces. 
Non-planar conformation of the isolated molecule of HBT (Fig. 2b) is predicted by DFTB calculations to have energy higher by $0.25 \mathrm{eV}$ than the most stable OH...N enol structure. The $\mathrm{N}_{7}-\mathrm{C}_{8}-\mathrm{C}_{10}-\mathrm{C}_{11}$ dihedral angle is $151^{\circ}$ in this isolated structure. Planarization of the structure by imposing $\mathrm{C}_{\mathrm{s}}$ symmetry restriction increases energy to $0.26 \mathrm{eV}$. Calculations [5] of structures of various conformations in the molecular cluster corresponding to the first coordination sphere predict that improper orientation of the benzothiazole fragment leads to the planarization of the structure and increase in energy by $0.69 \mathrm{eV}$.

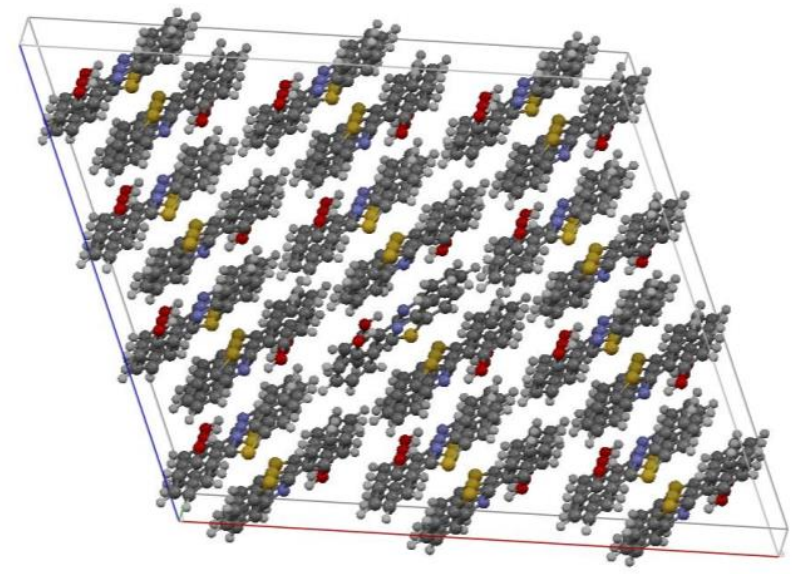

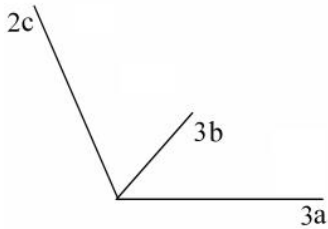

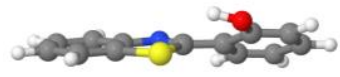

$\mathrm{b}$

Fig. 2. Unit cell of the periodic structure of HBT with a vacancy (a) and non-planar enol conformation of the molecule of HBT.

If the non-planar conformation is placed near the vacancy in the site of the molecule with the $\mathrm{N}_{7}-\mathrm{C}_{8}-\mathrm{C}_{10}-\mathrm{C}_{11}$ dihedral angle of $2.5^{\circ}$ then the structure remains non-planar. The $\mathrm{N}_{7}-\mathrm{C}_{8}-\mathrm{C}_{10}-\mathrm{C}_{11}$ dihedral angle in the molecule in the vicinity of the vacancy is $145^{\circ}$. The energy of the periodic structure increases by about $0.4 \mathrm{eV}$ that larger than the difference for isolated molecule but noticeable less than the value obtained for this molecular conformation in the rigid cluster corresponding to the ideal crystal lattice. Moreover, the non-planar conformation in the vicinity of the vacancy demonstrates larger deviation from the planarity than the isolated molecule.

\section{Conclusions}

Density-functional based tight-binding methods with Van der Waals interaction correction are used for modeling of vacancies in the molecular crystal of 2-(2'hydroxyphenyl)benzothiazole. The calculations are performed for periodic model structures corresponding to the arrangement of molecules in the crystal lattice.

The calculations demonstrate that absence of a molecule leads to deviations of the dihedral angles between benzothiazole and phenol fragments in the molecules in the vicinity of the vacancy from the values of the dihedral angles in the molecules in the bulk region of the modeled lattice. The vacancy is found to provide enough room for the nonplanar conformations of the molecule in the ground state.

Internal rotation in the excited state in the keto structure is considered to be a radiationless mechanism of transition to the ground state in the isolated molecules. Modeling of excited states in organic molecular crystals requires description of dispersion 
interaction in the excited states. The description of the dispersion forces is beyond the time dependent density-functional based tight-binding and even beyond the time dependent density functional theory methods. Van der Waals interaction correction used in our study involves atom-atom potential which parameters are valid for the ground state. The main drawback of the model is minimal size of region containing the defect needed for considering the vacancy. This implies regular arrangement of the defects in the model periodic structure.

\section{References}

1. Williams, D. L. Intramolecular proton transfer reactions in excited fluorescent compounds / D. L. Williams, A. Heller // J. Phys. Chem. - Vol. 74. - 1970. - P. 4473 4480 .

2. Pijeau, S. Excited-State Dynamics of 2-(2'-Hydroxyphenyl)benzothiazole: Ultrafast Proton Transfer and Internal Conversion / S. Pijeau, D. Foster, E. G. Hohenstein // J. Phys. Chem. A. - Vol. 121. - 2017. - P. 4595 - 4605.

3. Chang, S.M. Emission of white light from 2-(2'-hydroxyphenyl) benzothiazole in polymer electroluminescent devices / S.M. Chang, Y.J. Tzeng, S.Y. Wu, K.Y. Li, K.L. Hsueh / Thin Solid Films - 2005. - Vol. 447. - P. 38 - 41.

4. Zhang, W. Low-Threshold Wavelength-Switchable Organic Nanowire Lasers Based on Excited-State Intramolecular Proton Transfer / W. Zhang, Y. Yan, J. Gu, J. Yao, Y. S. Zhao // Angew. Chem. Int. Ed. - 2015. - Vol. 54. - P. 7125 - 7129.

5. Syetov, Y. Theoretical modeling of defects in the molecular crystal of 2-(2'hydroxyphenyl)benzothiazole / Y. Syetov // Journal of Physics and Electronics. - 2020.Vol. 28(1). - P. $43-48$.

6. Jia, A.-Q. Syntheses, characterization, and ethylene polymerization of titanium and zirconium complexes with [N, O] ligands / A.-Q. Jia and G.-X. Jin // Dalton Trans. 2009. - P. 8838-8845. CCDC 727487: Experimental Crystal Structure Determination. 2010. - DOI: $10.5517 /$ ccsf0bj.

7. Sténson, P. The Crystal Structure of 2-(o-Hydroxyphenyl)benzothiazole / P. Sténson // Acta Chem. Scand. - 1970. - Vol. 24. - P. 3729 - 3738.

8. Silinsh, E.A. Organic Molecular Crystals: Their Electronic States / E.A. Silinsh. // Springer Series in Solid-State Sciences. -Vol. 16. - Berlin Heidelberg: SpringerVerlag, 1980 - XVIII, 392 p.

9. Aradi, B. DFTB+, a Sparse Matrix-Based Implementation of the DFTB Method / B. Aradi, B. Hourahine, Th. Frauenheim // J. Phys. Chem. A. - Vol. 111. - 2007. - P. $5678-5684$.

10. Gaus, M. DFTB3: Extension of the Self-Consistent-Charge Density-Functional Tight-Binding Method (SCC-DFTB) / M. Gaus, Q. Cui, M. Elstner // J. of Chem. Theory Comput. - 2011. - Vol. 7. - P. $931-948$.

11. Syetov, Y. Low-frequency Raman spectrum of crystalline 2-(2'hydroxyphenyl)benzoxazole and density-functional based tight-binding phonon calculations / Y. Syetov // Ukr. J. Phys. Opt. - 2017. - Vol. 18, No. 2. - P. 67 - 76.

12. Schmidt, M. W. General Atomic and Molecular Electronic Structure System [Text] / M. W. Schmidt, K. K. Baldridge, J. A. Boatz, S. T. Elbert, M. S. Gordon, J. H. Jensen, S. Koseki, N. Matsunaga, K. A. Nguyen, S. J. Su, T. L. Windus, M. Dupuis, J. A. Montgomery // J. Comput. Chem. - 1993. - Vol. 14. - P. 1347 - 1363.

13. Gordon, M. C. Advances in electronic structure theory: GAMESS a decade later. / M. C. Gordon and M. W. Schmidt // Chapter 41 in Theory and Applications of Computational Chemistry, the first forty years. Ed. by Dykstra C. E., Frenking G., Kim K. S. and Scuseria G. E. - Elsevier: Amsterdam. - 2005. - p. 1167 - 1189.

84 